\title{
A Shallow Discourse Parsing System Based On Maximum Entropy Model
}

\author{
Jia Sun, Peijia Li, Weiqun Xu, Yonghong Yan \\ The Key Laboratory of Speech Acoustics and Content Understanding \\ Institute of Acoustics, Chinese Academy of Sciences \\ No. 21 North 4th Ring West Road, Haidian District, 100190 Beijing, China \\ \{sunjia, lipeijia, xuweiqun, yanyonghong\}@hccl.ioa.ac.cn
}

\begin{abstract}
This paper describes our system for Shallow Discourse Parsing - the CoNLL 2015 Shared Task. We regard this as a classification task and build a cascaded system based on Maximum Entropy to identify the discourse connective, the spans of two arguments and the sense of the discourse connective. We trained the cascaded models with a variety of features such as lexical and syntactic features. We also report the results achieved by our team.
\end{abstract}

\section{Introduction}

Discourse parsing is one of the most challenging tasks in natural language processing (NLP) field. It focuses on parsing the structure of a piece of text into a set of discourse relations between inter sentences. There is considerable interest in discourse parsing, both as an end in itself and as an intermediate step in a variety of NLP applications like question answering (Verberne et al., 2007), text summarization (Louis et al., 2010), sentiment analysis and opinion mining (Somasundaran, 2010).

There are many approaches working on identifying the discourse relations and data-driven approaches are dominated. A number of pioneers take the discourse relations identification as a classification task (Marcu and Echihabi, 2002; Pitler et al., 2009; Duverle and Prendinger, 2009) by the construction of features like lexical, syntactic and constituent features. Some take the argument segmentation task as a semantic role detection task (Wellner and Pustejovsky, 2007) and a sequence labeling task (Ghosh et al., 2011). However, some of the previous research is based on different corpus, lacking an common evaluation data set. This has been addressed with the release of Penn Discourse Treebank (PDTB) 2.0 corpus (Prasad et al.,
2008) which provides detailed annotations about the discourse relations and argument spans addresses this problem. Besides, much research about discourse parsing working on the PDTB appears (Prasad et al., 2010; Lin et al., 2009) and they put more attention on the "harder" part - labelling the arguments. Lin (Lin et al., 2014) designed an end-to-end discourse parser with the PDTB including the explicit, implicit sense and the argument spans identification.

Shallow Discourse Parsing (Xue et al., 2015) is the CoNLL shared task this year ${ }^{1}$ which takes a piece of newswire text as input and returns all the discourse relations in the form of a discourse connective (explicit or implicit) taking two arguments (which can be clauses, sentences, or multisentence segments) in JSON format. A relation will be parsed as correct if the explicit discourse connective (e.g., "because", "however") once it has, the spans of text that serve as the two arguments for each discourse connective and the sense (e.g., "Comparison") are all correct. The F1 score of the parser's performance is the evaluation metric.

In this paper, we describe our system details in Section 2, the evaluation result and subsequent experiments in Section 3. Finally, we draw some conclusions in Section 4.

\section{Our System}

\subsection{Resources}

The resources used in our system are as follows: Labeled training and development data: The training and development (dev) data is derived from the PDTB 2.0 Section 2-21 and Section 22 in JSON format. There are 32535 relations and 1436 relations annotated in the training data and the dev data respectively. Table 1 shows the distribution of the four types in the data. There are

\footnotetext{
${ }^{1}$ http://www.cs.brandeis.edu/ clp/conll15st/
} 


\begin{tabular}{|c|r|r|}
\hline Type & Train data & Dev data \\
\hline Explicit & 14,727 & 680 \\
Implicit & 13,163 & 522 \\
EntRel & 4,133 & 215 \\
AltLex & 524 & 19 \\
\hline all & 32,535 & 1,436 \\
\hline
\end{tabular}

Table 1: Distribution of the four discourse relation types in the data sets.

\begin{tabular}{|c|l|c|c|}
\hline Sense level 1 & Sense level 2or3 & Train & Dev \\
\hline \multirow{3}{*}{ Temporal } & A.P & 1,277 & 78 \\
& A.S & 1,014 & 55 \\
& Synchrony & 499 & 100 \\
\hline \multirow{5}{*}{ Contingency } & Cause.Reason & 3,344 & 147 \\
& Cause.Result & 2,137 & 81 \\
& Condition & 1,197 & 52 \\
\hline \multirow{5}{*}{ Comparison } & Contrast & 4,714 & 257 \\
& Concession & 1,293 & 17 \\
\hline & Conjunction & 7,817 & 310 \\
& Instantiation & 1,403 & 58 \\
& Restatement & 2,699 & 110 \\
& Alternative & 210 & 6 \\
& A.C & 241 & 6 \\
& Exception & 15 & 0 \\
\hline & EntRel & 4,133 & 215 \\
\hline
\end{tabular}

Table 2: Distribution of the 15 senses from the different data sets. A.P, A.S, A.C are the abbreviations of "Asynchronous.Precedence", "Asynchronous.Succession", "Alternative.Chosen alternative" respectively .

15 valid senses including the second-level "types" as well as a selected number of third-level "subtypes". Table 2 shows the distribution of the 15 senses in the data.

Test data: There are two test data sets. One is the blind set which contains 20,000 to 30,000 words of newswire text annotated following the PDTB annotation guidelines. The other test set is Section 23 of the PDTB which is used for comparison with previous work.

The connectives list: A list contains 100 discourse connectives in the PDTB and three syntactic categories form (Knott, 1996).

Opennlp-maxent: We used the open source package Opennlp-maxent ${ }^{2}$ to construct the classification models.

\footnotetext{
${ }^{2}$ http://sourceforge.net/projects/maxent/files/
}

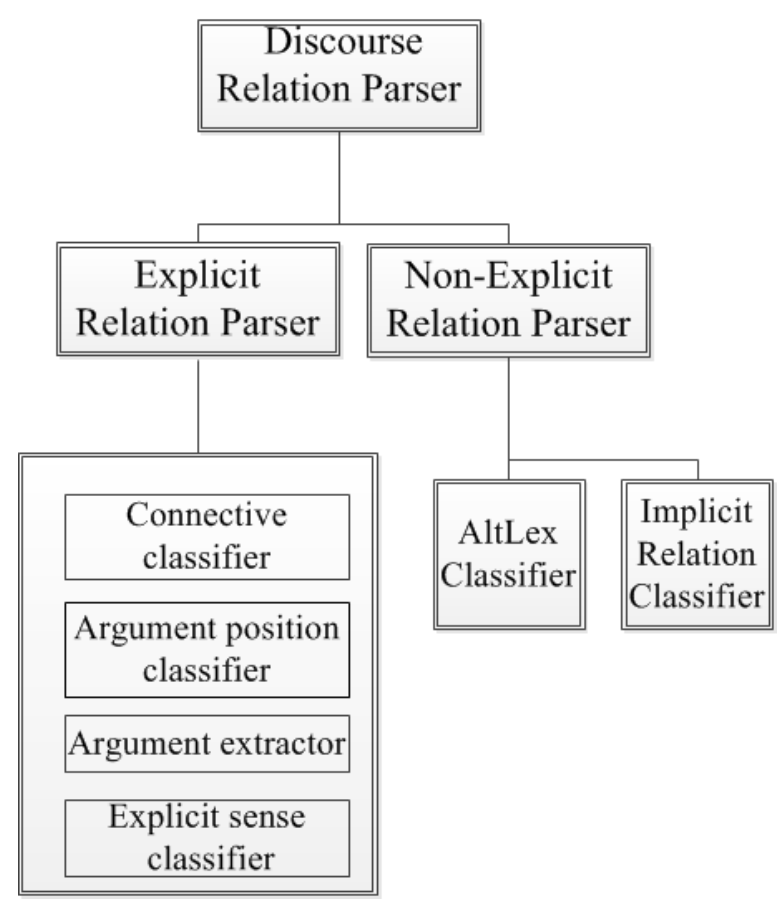

Figure 1: The structure of the system.

\subsection{System overview and Features}

Our system mainly follows the work of (Lin et al., 2014), which consists of two parts: the explicit relation parser and the non-explicit relation parser. The explicit relation parser is composed of the connective classifier, the argument position classifier, the argument extractor and the explicit sense classifier while the non-Explicit relation parser contains the AltLex classifier and the implicit classifier. The structure of our system is shown in Figure 1.

The set of features used in our system are listed in Table 3. All the features fall into four classes: lexical features, part-of-speech (POS) features, syntactic features and positional features.

- Lexical features: The lexical features (F1F10) contain the connectives C, their contextual words and word-pair features (i.e., F7 $\left(w_{i}, w_{j}\right)$ where $w_{i}$ is a word from $\operatorname{Arg} 1$ and $w_{j}$ is a word from $\left.\operatorname{Arg} 2\right)$.

- POS features: F11-F17 belong to the POS features .

- Syntactic features: The syntactic features (F18-F26) include the connectives' syntactic category (F18): subordinating, coordinating, or discourse adverbial, the path of syntactic trees (F19, F20, F23), the number of siblings 


\begin{tabular}{|c|c|}
\hline Feature & Description \\
\hline F1. & C string \\
\hline $\mathrm{F} 2$. & the first word before $\mathrm{C}$ \\
\hline F3. & the second word before $\mathrm{C}$ \\
\hline F4. & $\mathrm{F} 2+\mathrm{C}$ \\
\hline F5. & $\mathrm{F} 3+\mathrm{C}$ \\
\hline F6. & $\mathrm{C}+$ the next word after $\mathrm{C}$ \\
\hline F7. & word-pair \\
\hline F8. & the first word of Arg2 \\
\hline F9. & the second word of $\operatorname{Arg} 2$ \\
\hline F10. & the third word of $\operatorname{Arg} 2$ \\
\hline F11. & the POS of C \\
\hline F12. & the POS of F2 \\
\hline F13. & $F 11+F 12$ \\
\hline F14. & the POS of the word after C \\
\hline F15. & $\mathrm{F} 11+\mathrm{F} 14$ \\
\hline F16. & the POS of F3 \\
\hline F17. & $\mathrm{F} 11+\mathrm{F} 16$ \\
\hline F18. & the syntactic category of $\mathrm{C}$ \\
\hline F19. & the path of C's parent to root \\
\hline F20. & the compressed path of F19 \\
\hline F21. & the number of left siblings of $\mathrm{C}$ \\
\hline F22. & the number of right siblings of $\mathrm{C}$ \\
\hline F23. & the path of C's parent to $\mathrm{N}$ \\
\hline F24. & $\begin{array}{l}\text { whether the C's left sibling number } \\
\text { is greater than } 1\end{array}$ \\
\hline F25. & the constituent rules \\
\hline F26. & the dependency rules \\
\hline F27. & the relative position of $\mathrm{N}$ to $\mathrm{C}$ \\
\hline F28. & the position of $\mathrm{C}$ in the sentence \\
\hline
\end{tabular}

Table 3: The features used in our system. "C" denotes the connectives. $\mathrm{N}$ means a current node in the constituent tree used in Section 2.3.2.

(F21, F22, F24), constituent rules (F25) and dependency rules (F26).

- Position features: F27 is the relative position in the syntactic tree structure (left, middle or right), while F28 is the connectives' positions in the sentence (start, middle or end).

\subsection{Training}

\subsubsection{The Connective Classifier}

All the 100 connectives that appeared in one discourse were extracted whether it functioned as a connective or not. We converted all upper case letters in connective to lower case ones.

The connective classifier decides whether a connective is functioned as a discourse connective.
The features used were F4, F6, F11-F15, F19-F20 in Table 3.

\subsubsection{The Argument Labeller}

Once the connective is identified, the argument labeller identifies the Arg1 and Arg2 spans of this instance. This is accomplished in two steps: (1) Classifying the locations of Arg1 by the Argument Position Classifier. (2) Labelling the spans of Arg1 and Arg2 by the Argument Extrator.

The Argument Position Classifier: Normally Arg2 immediately follows the connective while the position of $\operatorname{Arg} 1$ is uncertain. In this model, we classified the Arg1's locations into two classes: Arg1 was located within the same sentence of the connective (SS) or in the previous sentence of connective (PS) (Prasad et al., 2008).

We implemented this as a binary classification task. In this step, features F1-F5, F11, F13, F16F17, F28 in Table 3 were adopted to train the model. After the position label of Arg1 was determined, the result was passed to the argument extractor.

The Argument Extractor: In this module, our classifier labelled the previous sentence as Arg1 immediately for the PS case. The argument spans for the SS case were extracted described as below.

- Classify each internal node $\mathrm{N}$ in the constituent tree as Arg1-node, Arg2-node, or None with features F1, F18, F21-F24, F27 in Table 3.

- Label a node as Arg1-node once its Arg1node predicted probability is greater than 0.1 (which is tuned on the dev data set).

- Select only one Arg1-node and one Arg2node in one instance with the maximal probability of the respective label.

- Extract the Arg1 and Arg2 spans by tree subtraction. If the $\operatorname{Arg} 1$ node is the ancestor of the Arg2 node, the span of Arg1 should be subtracted from the Arg2 span, and vice versa.

- Remove punctuation tokens and connectives out of the exact argument spans.

\subsubsection{The Explicit Sense Classifier}

After recognizing the discourse connective and its two arguments spans, the next step is to decide the 


\begin{tabular}{|c|c|c|c|c|}
\hline Data & Connective & Span & Sense & Parser \\
\hline Dev & 0.9152 & 0.2668 & 0.1367 & 0.1814 \\
\hline Test & 0.9064 & 0.2336 & 0.1191 & 0.1505 \\
\hline Blind & 0.826 & 0.2195 & 0.1232 & 0.1262 \\
\hline
\end{tabular}

Table 4: The results F1 score obtained by our team

sense of the connective. We trained this model using features F1, F4, F11 in Table 3. We picked the output whose maximal sense probability is greater than 0.45 which was experientially determined on dev data set.

\subsubsection{The AltLex classifier}

We extracted all adjacent sentence pairs within each paragraph and removed the pairs that were identified by the explicit relation parser. Then we trained the AltLex Classifier which decided whether the pairs were AltLex pairs and classified the senses with features F8-F10 in Table 3. The pairs labelled as non-AltLex relations were passed to the next implicit relation classifier.

\subsubsection{The Implicit relation classifier}

The implicit relation classifier classified the sense of each pair into one of the 15 valid senses or NoRel with F7, F25-F26 in Table 3. After predicting, we kept the implicit discourse relations whose maximal sense probability were greater than a threshold ( 0.25 in our case) which was determined on the dev data set .

\section{Experiments and Results}

There are two test data sets this year as described in Section 2.1 and the organizers reported the results on the two test data sets and the dev data set. The results of our system obtained are shown in Table 4. We ranked the 10th on every data set.

After the deadline of evaluation, we made some improvements in the module of implicit relation classifier inspired by (Lin et al., 2009). We selected the word-pair features (F7) while the experiments showed a little degradation in F1 score through selecting the constituent rules and the dependency rules (F25, F26) on the dev data set.

We computed the mutual information between each word-pair feature and the 15 valid senses and then selected the top $\mathrm{N}$ as the features. Table 5 shows the improvement of different $\mathrm{N}$.

\begin{tabular}{|c|c|c|}
\hline $\mathbf{N}$ & Non-Explicit & Overall \\
\hline 50 & 0.0683 & 0.1876 \\
100 & 0.0683 & 0.1876 \\
200 & 0.0614 & 0.1870 \\
300 & 0.0603 & 0.1870 \\
\hline Baseline & 0.0435 & 0.1814 \\
\hline
\end{tabular}

Table 5: The F1 score in non-explict and overall parser when selecting features F7 using different $\mathrm{N}$ on dev data set.

\section{Conclusion}

We divided the complex task of discourse parsing into a set of classification subtasks and glued them together. A variety of features, including lexical, part-of-speech, syntactic and positional feature were employed to train the baseline with open Maximum Entropy package, then the system was improved by setting probability-output threshold. We did not utilize any additional resources and only used the annotations the official provided. Our system ranked the 10th among seventeenth teams on the two test data sets.

\section{Acknowledgments}

We would like to thank the shared task organizers for their support throughout this work. This work is partially supported by the National Natural Science Foundation of China (Nos. 11161140319, 91120001, 61271426), the Strategic Priority Research Program of the Chinese Academy of Sciences (Grant Nos. XDA06030100, XDA06030500), the National 863 Program (No. 2012AA012503) and the CAS Priority Deployment Project (No. KGZD-EW103-2).

\section{References}

David A Duverle and Helmut Prendinger. 2009. A novel discourse parser based on support vector machine classification. In Proceedings of the Joint Conference of the 47th Annual Meeting of the ACL and the 4th International Joint Conference on Natural Language Processing of the AFNLP: Volume 2Volume 2, pages 665-673. Association for Computational Linguistics.

Sucheta Ghosh, Richard Johansson, and Sara Tonelli. 2011. Shallow discourse parsing with conditional random fields. In In Proceedings of the 5th International Joint Conference on Natural Language Processing (IJCNLP 2011). 
Alistair Knott. 1996. A data-driven methodology for motivating a set of coherence relations.

Ziheng Lin, Min-Yen Kan, and Hwee Tou Ng. 2009. Recognizing implicit discourse relations in the penn discourse treebank. In Proceedings of the 2009 Conference on Empirical Methods in Natural Language Processing: Volume 1-Volume 1, pages 343-351. Association for Computational Linguistics.

Ziheng Lin, Hwee Tou Ng, and Min-Yen Kan. 2014. A pdtb-styled end-to-end discourse parser. Natural Language Engineering, 20(2):151-184.

Annie Louis, Aravind Joshi, and Ani Nenkova. 2010. Discourse indicators for content selection in summarization. In Proceedings of the 11th Annual Meeting of the Special Interest Group on Discourse and Dialogue, pages 147-156. Association for Computational Linguistics.

Daniel Marcu and Abdessamad Echihabi. 2002. An unsupervised approach to recognizing discourse relations. In Proceedings of the 40th Annual Meeting on Association for Computational Linguistics, pages 368-375. Association for Computational Linguistics.

Emily Pitler, Annie Louis, and Ani Nenkova. 2009. Automatic sense prediction for implicit discourse relations in text. In Proceedings of the Joint Conference of the 47th Annual Meeting of the ACL and the 4th International Joint Conference on Natural Language Processing of the AFNLP: Volume 2-Volume 2, pages 683-691. Association for Computational Linguistics.

Rashmi Prasad, Nikhil Dinesh, Alan Lee, Eleni Miltsakaki, Livio Robaldo, Aravind K. Joshi, and Bonnie L. Webber. 2008. The penn discourse treebank 2.0. In Proceedings of the International Conference on Language Resources and Evaluation, LREC 2008, 26 May - 1 June 2008, Marrakech, Morocco.

Rashmi Prasad, Aravind K. Joshi, and Bonnie L. Webber. 2010. Exploiting scope for shallow discourse parsing. In Proceedings of the International Conference on Language Resources and Evaluation, LREC 2010, 17-23 May 2010, Valletta, Malta.

Swapna Somasundaran. 2010. Discourse-level relations for Opinion Analysis. Ph.D. thesis, University of Pittsburgh.

Suzan Verberne, Lou Boves, Nelleke Oostdijk, and Peter-Arno Coppen. 2007. Evaluating discoursebased answer extraction for why-question answering. In Proceedings of the 30th annual international ACM SIGIR conference on Research and development in information retrieval, pages 735-736. ACM.

Ben Wellner and James Pustejovsky. 2007. Automatically identifying the arguments of discourse connectives. In Proceedings of the 2007 Joint Conference on Empirical Methods in Natural Language Processing and Coumptational Natural Language Learning (EMNLP-CoNLL), pages 92-101.
Nianwen Xue, Hwee Tou Ng, Sameer Pradhan, Rashmi Prasad, Christopher Bryant, and Attapol Rutherford. 2015. The CoNLL-2015 shared task on shallow discourse parsing. In Proceedings of the Nineteenth Conference on Computational Natural Language Learning. 